\title{
The Impact of Disease Severity in Carpal Tunnel Syndrome on Grip Strength, Pinch Strength, Fine Motor Skill and Depression
}

\author{
Nilgun Simsir Atalay, MD, $\mathrm{PhD}^{1)}$, Ayse SARSAN, MD, $\mathrm{PhD}^{1)}$, \\ NuRAY AKKAYA, MD, PhD ${ }^{1)}$, NeCMETTIN YILDIZ, MD, PhD $^{1)}$, OYA TOPUZ MD, PhD ${ }^{1)}$ \\ 1) Pamukkale University Medical School, Department of Physical Medicine and Rehabilitation: \\ 20210, Kinikli / Denizli, Turkey. TEL: +90 258-211-81-29, FAX: +90 258-211-81-29, \\ E-mail:drnilgunatalay@gmail.com
}

\begin{abstract}
Purpose] We evaluated the impact of disease severity on symptoms, functional status of hand and depression of patients diagnosed as having carpal tunnel syndrome (CTS). [Subjects and Methods] Ninety-nine patients with diagnosis of unilateral CTS in the dominant extremity were enrolled in the study. Patients' clinical parameters, grip strength, lateral, tip and tripod pinch strengths, two-point discrimination (2-PD), Semmes Weinstein Monofilament test and Purdue Pegboard test (PPT) were evaluated. The Beck Depression Scale (BDS) was used to evaluate patients. The patients were divided into 3 groups, mild, moderate and advanced CTS, according to electrophysiological parameters. [Results] Thirty-three patients had mild, 34 patients had moderate and 32 patients had advanced CTS. There were statistically significant decreases in tripod and tip pinch strength and a statistically significant increase in the score of the 2-PD test in the advanced CTS group compared to the mild and moderate CTS groups. While there was a significant decrease in the dominant hand subtest of PPT in the advanced CTS group compared to the mild CTS group, no differences in the other subtests of PPT were found. The BDS scores of patients with advanced CTS were significantly higher than those of patients with mild CTS. [Conclusion] Tip and tripod pinch strength decreased, sense of touch and fine motor skill deteriorated and depression status increased with disease severity.
\end{abstract}

Key words: Carpal Tunnel Syndrome, Pinch strength, Depression

(This article was submitted Jul. 19, 2010, and was accepted Aug. 19, 2010)

\section{INTRODUCTION}

Carpal tunnel syndrome (CTS) is the most common entrapment neuropathy in the upper limbs and involves compression of the median nerve at the wrist $^{1-4)}$. The incidence and prevalence varies, $0.125 \%-1 \%$ and $5 \%-16 \%$, depending upon the diagnostic criteria used ${ }^{5-11)}$. Carpal tunnel syndrome is characterized by burning pain associated with tingling and numbness in the distribution of the median nerve distal to the wrist and weakness in the thenar muscles, decrease in hand function and skills, and can cause psychological symptoms in advanced cases ${ }^{12-15)}$. Median nerve conduction studies are $49 \%-84 \%$ sensitive and $95 \%$ $99 \%$ specific and are accepted as the gold standard for diagnosis $^{12)}$.

Various results have been reported for associations between electrodiagnostic findings and clinical symptoms. While some studies have reported no association between electrodiagnostic findings and severity of clinical symptoms ${ }^{16-18)}$, others have determined positive correlations $^{19,20)}$.

In many studies, parameters such as hand grip strength, fine motor skills and psychological condition have been used to determine the efficacy of therapies for carpal tunnel syndrome, but few studies have investigated the associations between these parameters and electrodiagnostic findings ${ }^{21,22)}$. Moreover, studies about depression levels and severity of CTS are few in number ${ }^{22)}$.

We aimed to assess the influence of disease severity determined by electrodiagnosis on grip strength, pinch strength, fine motor skill and depression in carpal tunnel syndrome.

\section{SUBJECTS AND METHODS}

The study was conducted at the outpatient clinic of Pamukkale University, Department of Physical Medicine and Rehabilitation. Ninety-nine patients diagnosed with unilateral carpal tunnel syndrome in the dominant extremity were enrolled in the study.

All subjects were interviewed for age, gender, duration of symptoms, concomitant diseases, dominant extremity, symptoms of CTS (paresthesia, pain, awoken paresthesia) and were examined using the Tinel, Phalen, and reverse 
Phalen tests. The Tinel test is assumed positive when tapping the median nerve along its course in the wrist worsens the tingling in the distribution of median nerve. The Phalen test is regarded as positive when holding both wrists in complete and forced flexion for 1 minute causes symptoms in the distribution of the median nerve. The reverse Phalen test is accepted positive when maintaining the fingers and wrists in full extension for 1 minute causes the symptoms.

Subjects were excluded from the study if they had diabetes mellitus, acromegaly, hypothyroidia, kidney or connective tissue disease, malignancy, cervical disc disease, lateral epicondylitis, tenosynovitis of DeQuervein, trigger finger, fibromyalgia, history of distal radial fracture, or a history of surgery for CTS.

Nerve conduction studies were performed at room temperature, $25^{\circ} \mathrm{C}$, with four electrode electroneuromyography (ENMG) devices (Medelec Synergy) while patients were lying supine. Bilateral median motor nerve distal latencies, median motor nerve conduction velocities, median sensory nerve conduction velocities from the wrist to second digit and wrist to palm, and motor and sensory action potential amplitudes were measured. Bilateral ulnar nerve sensory and motor conduction studies were performed to exclude any possible diseases. The severity of carpal tunnel syndrome was classified as mild, moderate, or advanced according to the American Association of Electrodiagnostic Medicine guidelines ${ }^{23}$.

Assesment of hand grip strength was evaluated with a Jamar dynamometer. Measurements were taken while the patients were sitting with the elbow flexed at $90^{\circ}$, and with the forearm and wrist in the neutral position ${ }^{24)}$. The mean of three measurements was used in the analysis.

Pinch grip strength was measured in the dominant extremity by pinchmeter in three different positions for lateral, tip and tripod pinches. Three measurements were made and the mean value was used ${ }^{24)}$.

Sense of touch was evaluated by the Static 2 Points Discrimination test (2-PD) and the Semmes Weinstein Monofilament test (SWMT) using 2.83, 3.61 and 4.31 filaments ${ }^{24)}$. The mean values of the first three digits of the dominant extremity were calculated.

The Purdue Pegboard Test (PPT) was used for fine motor activity evaluation. It consists of four subtests: 1 - Fine grip of dominant hand (PPT 1) 2- Fine grip of non-dominant hand (PPT 2) 3- Fine grip of bilateral hands (PPT 3) 4Assembly of both hands (PPT 4) ${ }^{25)}$.

The depression state of patients was evaluated using the Beck Depression Scale (BDS). BDS is a self-report instrument for assessing the severity of depression. It is composed of 21 items. Scores from 0-9 represent minimal depressive symptoms, 10-18 mild, 19-29 moderate, 30-63 indicate severe depression ${ }^{22)}$. The validity of BDS for the Turkish population was shown by Hisli, et al. ${ }^{26)}$.

Statistical analysis were performed using SPSS 17.0 for Windows. The demographic and clinical characteristics of patients were evaluated with descriptive statics. KruskallWallis variance analysis was used for comparisons between groups. When a difference between groups was found, the
Table 1. The demographic data of the patients

\begin{tabular}{ll}
\hline & Patients with CTS \\
\hline Age (years) (mean \pm SD) & $49.7 \pm 8.4$ \\
Females / Males n (\%) & $70(70.7) / 29(29.3)$ \\
Dominant hand (right) n (\%) & $98(99)$ \\
$($ left $) \mathrm{n}(\%)$ & $1(1)$ \\
BMI $\left(\mathrm{kg} / \mathrm{m}^{2}\right)($ mean $\pm \mathrm{SD})$ & $28.8 \pm 4.3$ \\
Disease duration (months) (mean $\pm \mathrm{SD})$ & $9.47 \pm 6.17$
\end{tabular}

SD: Standard deviation.

Mann-Whitney $U$ test was used to determine in which group the difference occurred; values of $\mathrm{p}<0.05$ were considered statistically significant.

\section{RESULTS}

In this study ninety-nine patients with unilateral CTS in the dominant extremity were evaluated. The demographic data are summarized in Table 1. The Tinel test was positive in $72(72.7 \%)$ patients, the Phalen test was positive in 77 $(77.8 \%)$ patients, and the reverse Phalen test was positive in $74(74.7 \%)$ patients.

Thirty-three patients $(33.3 \%)$ had mild, 34 patients $(34.3 \%)$ had moderate, and 32 patients $(32.3 \%)$ had advanced CTS according to the electrodiagnostic results for the dominant extremity. The demographic and clinical characteristics of the patients according to severity of CTS are summarized in Table 2.

There were no statistically significant differences between groups regarding age, BMI, duration of disease, and positivity of clinical parameters $(p>0.05)$. The comparison of clinical parameters between CTS groups is summarized in Table 3.

In the comparison of motor functions, there were statistically significant decreases in tripod and tip pinch strength between the advanced CTS group and the mild and moderate CTS groups $(\mathrm{p}<0.05)$.

In the evaluation of sensory function, there was a statistically significant increase in the 2-PD test in the advanced CTS group compared to the mild and moderate CTS groups $(\mathrm{p}<0.05)$.

In the PPT, which evaluates the fine motor function, a statistically significant decrease in the advanced CTS group in respect to mild CTS group was found only in the subtests of dominant hand $(\mathrm{p}<0.05)$, and there were no differences in the other tests $(p>0.05)$.

BDS scores of the advanced CTS group were significantly higher than those of the mild CTS group $(\mathrm{p}<0.05)$.

\section{DISCUSSION}

Many studies have reported that peak incidence of CTS is at 55-60 years of life, that women are affected more than men, and that the right hand is affected more than the left hand $^{12,27)}$. In our study, mean age and gender of the patients were consistent with those reported in the literature. The 
Table 2. Comparison of demographic characteristics and clinical findings

\begin{tabular}{lccc}
\hline & Mild CTS $(\mathrm{n}=33)$ & Moderate CTS $(\mathrm{n}=34)$ & Advanced CTS $(\mathrm{n}=32)$ \\
\hline Age (years) (mean \pm SD) & $49.3 \pm 7.6$ & $50.3 \pm 10.4$ & $49.6 \pm 6.8$ \\
BMI (kg/m ${ }^{2}$ ) (mean \pm SD) & $28.6 \pm 3.2$ & $29.5 \pm 5.1$ & $28.1 \pm 4.2$ \\
Disease duration (months) (mean \pm SD) & $8.87 \pm 4.83$ & $10.50 \pm 7.02$ & $9.00 \pm 6.50$ \\
Phalen test n (\%) & $26(78.8)$ & $27(79.4)$ & $27(84.4)$ \\
Tinel test $\mathrm{n}(\%)$ & $25(75.8)$ & $26(76.5)$ & $27(84.4)$ \\
Reverse Phalen test n (\%) & $26(78.8)$ & $28(82.4)$ & $28(87.5)$ \\
Paresthesia n (\%) & $26(78.8)$ & $26(76.5)$ & $29(90.6)$ \\
Nocturnal paresthesia n (\%) & $29(87.9)$ & $32(94.1)$ & $31(96.9)$ \\
Pain n (\%) & $21(63.6)$ & $18(52.9)$ & $22(68.8)$ \\
\hline
\end{tabular}

SD: Standard deviation.

Table 3. Comparison of clinical parameters and results of BDS scores in the patient groups

\begin{tabular}{lccc}
\hline & Mild CTS $(\mathrm{n}=33)$ & Moderate CTS $(\mathrm{n}=34)$ & Advanced CTS $(\mathrm{n}=32)$ \\
\hline Grip strength $(\mathrm{kg})$ & $22.78 \pm 2.58$ & $22.44 \pm 2.32$ & $21.97 \pm 2.18$ \\
Lateral pinch strength $(\mathrm{kg})$ & $7.37 \pm 0.78$ & $7.16 \pm 0.65$ & $7.03 \pm 0.88$ \\
Tripod pinch strength $(\mathrm{kg})$ & $6.86 \pm 0.63^{*}$ & $6.54 \pm 0.63^{*}$ & $4.57 \pm 0.48^{*}$ \\
Tip pinch strength $(\mathrm{kg})$ & $6.60 \pm 0.93^{*}$ & $6.47 \pm 0.88^{*}$ & $4.13 \pm 0.56^{*}$ \\
2-PD test & $5.11 \pm 0.28^{*}$ & $5.20 \pm 0.33^{*}$ & $5.85 \pm 0.84^{*}$ \\
SWMT & $3.33 \pm 0.31$ & $3.38 \pm 0.23$ & $3.45 \pm 0.26$ \\
PPT 1 & $11.72 \pm 1.79^{*}$ & $10.85 \pm 1.67$ & $10.31 \pm 1.71^{*}$ \\
PPT 2 & $13.12 \pm 1.59$ & $12.32 \pm 1.75$ & $12.53 \pm 1.77$ \\
PPT 3 & $17.69 \pm 2.88$ & $17.14 \pm 2.77$ & $16.56 \pm 3.21$ \\
PPT 4 & $4.60 \pm 1.05$ & $4.44 \pm 0.92$ & $4.12 \pm 0.94$ \\
BDS & $7.36 \pm 1.91^{*}$ & $8.17 \pm 1.60$ & $8.87 \pm 1.62^{*}$ \\
\hline
\end{tabular}

$* \mathrm{p}<0.05$.

mean age and the gender percentage not differ between the groups when patients were grouped according to severity of CTS.

In our study, we compared motor and sensory functions, fine motor skills and depression levels of patients with unilateral CTS, classified as mild, moderate or advanced, using electrodiagnostic analysis.

It was reported that the weakness of abductor pollicis brevis and opponens pollicis might be compensated by synergistic muscles in CTS, therefore grip strength and lateral pinch strength are not affected significantly. Tip and tripod pinch strength rely more on the thenar muscles and are therefore more suitable measures of motor involvement in $\mathrm{CTS}^{28)}$. In our study, while there were no differences in grip strength or lateral pinch strength with respect to disease severity, decreases in tip and tripod pinch strength were seen in the mild, moderate and advanced CTS groups respectively, consistent with reports in the literature. Similarly Keskin, et al. ${ }^{21)}$ reported that tripod pinch strength was decreased in these three groups.

In this study, 2-PD and SWMT were used to evaluate the sensory function of patients. Katz, et al. ${ }^{29)}$ reported that 2PD assesses median nerve dysfunction directly and served as a standard tool for assessing the results of carpal tunnel surgery. While we found statistically significant differences between advanced and moderate CTS $(p<0.05)$, and between advanced and mild CTS $(\mathrm{p}<0.05)$ for $2-\mathrm{PD}$, we did not find any significant difference among the groups for SWMT ( $>00.05)$. Pagel, et al. ${ }^{30)}$ suggested that SWMT has no utility in diagnosing electrodiagnostically confirmed CTS patients. Contrary to our results, Keskin, et al. ${ }^{21)}$ reported that values of SWMT were significantly higher in advanced CTS patients than in mild or moderate CTS patients.

Different subtests of PPT are used for functional evaluation of fine motor control of both hands in $\mathrm{CTS}^{31,32)}$. In the literature, twenty patients with unilateral moderate CTS, symptom duration of 2.7 years, and twenty healthy controls were compared for fine motor control activity using PPT. Patients with CTS had bilateral deficiencies in four subtests of PPT with respect to healthy controls, and bilateral deficiencies in fine motor control and tip pinch strength were found to negatively correlate with symptom duration and intensity of pain ${ }^{32}$ ). In our study, while there was a significantly decrease in the subtest of PPT of the dominant hand in the advanced CTS group compared to the mild CTS group $(p<0.05)$, there were no differences in the other subtests of PPT $(\mathrm{p}>0.05)$. We suggest that this situation is related to symptom duration. The mean symptom duration in our study was $9.47 \pm 6.17$ months, which was considerably shorter than the symptom duration reported in the study cited above.

Chan, et al. ${ }^{16)}$ found that depression scores of patients with CTS were 1-1.5 standard deviation greater than those of healthy controls. Similarly Tekeoglu, et al. ${ }^{22}$ found that depression scores were higher in patients with CTS than in controls. However, in this study, there was no significant association between the intensity of ENMG and BDS 
( $>0.05$ ), although we did find that BDS scores in the advanced CTS group were significantly higher than those in the mild CTS group $(\mathrm{p}<0.05)$. The limitations of this study are absence of a control group and not knowing the concomitant other psychiatric diseases of the patients.

In conclusion tip and tripod pinch strength, sensation of touch and fine motor activity of the hand are affected in direct proportion to the severity of CTS, and depression levels worsen with severity of CTS. These factors should be taken into consideration in the diagnosis and treatment of advanced CTS patients. Further research is needed to explore this situation with controlled randomised studies with more participants.

\section{REFERENCES}

1) Hadler NM: Nevre entrapment syndromes. In: McCarty DJ, Kopman WJ (ed) Arthritis and allied conditions. Lea and Febiger: Philadelphia, 1993, pp 16191624.

2) Omer GE Jr: Median nerve compression at the wrist. Hand Clin, 1992, 8: $317-$ 324.

3) Patterson JD, Simmons BP: Outcomes assessment in carpal tunnel syndrome. Hand Clin, 2002, 18: 359-363.

4) Katz JN, Simmons BP: Clinical practice. Carpal tunnel syndrome. N Engl J Med, 2002, 346: 1807-1812.

5) Tanaka S, Wild DK, Seligman PJ, et al.: The US prevalence of self-reported carpal tunnel syndrome: 1988 National Health Interview Survey data. Am J Public Health, 1994, 84: 1846-1848.

6) Atroshi I, Gummesson C, Johnsson R, et al.: Prevalence of carpal tunnel syndrome in a general population. JAMA, 1999, 282: 153-158.

7) de Krom MC, Knipschild PG, Kester AD, et al.: Carpal tunnel syndrome: prevalence in the general population. J Clin Epidemiol, 1992, 45: 373-376.

8) Stevens JC, Sun S, Beard CM, et al.: Carpal tunnel syndrome in Rochester, Minnesota, 1961 to 1980 . Neurology, 1988, 38: 134-138.

9) Einhorn N, Leddy JP: Pitfalls of endoscopic carpal tunnel release. Orthop Clin North Am, 1996, 27: 373-380.

10) Ferry S, Pritchard T, Kenan J, et al.: Estimating the prevalence of delayed median nevre conduction in the general population. Br J Rheumatol, 1998, 37 : 630-635.

11) Prick JJ, Blaauw G, Vredeveld JW, et al.: Results of carpal tunnel release. Eur J Neurol, 2003, 10: 733-736.

12) Aroori S, Spence RA: Carpal tunnel syndrome. Ulster Med J, 2008, 77: 6-17.

13) Muller M, Tsui D, Schnurr R, et al.: Effectiveness of hand therapy interventions in primary management of carpal tunnel syndrome: a systematic review. J Hand Ther, 2004, 17: 210-218.

14) Gupta SK, Bestead TJ: Symptoms experienced by patients with carpal tunnel syndrome. Can J Neurol Sci, 1997, 24: 338-342.

15) Atroshi I, Johnsson R, Nouhan R, et al.: Use of outcome instruments to compare workers' compensation and non-workers' compensation carpal tunnel syndrome. J Hand Surg Am, 1997, 22: 882-888.

16) Chan L, Turner JA, Comstock BA, et al.: The relationship between electrodiagnostic findings and patient symptoms and function in carpal tunnel syndrome. Arch Phys Med Rehabil, 2007, 88: 19-24

17) Schrijver HM, Gerritsen AA, Strijers RL, et al.: Correlating nerve conduction studies and clinical outcome measures on carpal tunnel syndrome: lessons from a randomized controlled trial. J Clin Neurophysiol, 2005, 22: 216-221.

18) Longstaff L, Milner RH, O'Sullivan S, et al.: Carpal tunnel syndrome: the correlation between outcome, symptoms and nerve conduction study findings. J Hand Surg Br, 2001, 26: 475-480.

19) You H, Simmons Z, Freivalds A, et al.: Relationships between clinical symptom severity scales and nerve conduction measures in carpal tunnel syndrome. Muscle Nerve, 1999, 22: 497-501.

20) Dhong ES, Han SK, Lee BI, et al.: Correlation of electrodiagnostic findings with subjective symptoms in carpal tunnel syndrome. Ann Plast Surg, 2000, 45: 127-131.

21) Keskin D, Uçan H, Babaoglu S, et al.: Evaluation of clinical, electromyographic parameters and quality of life in patients with carpal tunnel syndrome. Turkiye Klinikleri J Med Sci, 2008, 28: 456-461.

22) Tekeoglu I, Gulcu E, Sayin R, et al.: Sleep quality, depression and anxiety in carpal tunnel syndrome. Turk J Phys Med Rehab, 2008, 54: 102-106.

23) Stevens JC: AAEM minimonograph \#26: the electrodiagnosis of carpal tunnel syndrome. American Association of Electrodiagnostic Medicine. Muscle Nevre, 1997, 20: 1477-1486.

24) Aulicino PL: Clinical examination of the hand. In: Hunter $M$ (ed) Rehabilitation of the hand and upper extremity, 5th edn) St Louis, Mosby, 2002, pp120-142.

25) Dilek B, Gulbahar S, Bacakoglu K, et al.: Rehabilitation outcomes after upper extremity replantation. Turk J Phys Med Rehab, 2009, 55: 98-101.

26) Hisli N: Beck Depresyon Envanteri'nin geçrliligi üzerine bir çaliș ma. Turkish Journal of Psychology, 1988, 6: 118-122.

27) Padua L, LoMonaco M,Gregori, B, et al.: Neurophysiological classification and sensitivity in 500 carpal tunnel syndrome hands. Acta Neurol Scand, 1997, 96: 211-217.

28) Geere J, Chester R, Kale S, et al.: Power grip, pinch grip, manual muscle testing or thenar atrophy-which should be assessed as a motor outcome after carpal tunnel decompressions? Asystematic review. BMC Musculoskelet Disord, 2007, 8: 114.

29) Katz JN, Gelberman RH, Wright EA, et al.: Responsiveness of self-reported and objective measures of disease severity in carpal tunnel syndrome. Med Care, 1994, 32: 1127-1133.

30) Pagel KJ, Kaul MP, Dryden JD: Lack of utility of Semmes-Weinstein monofilament testing in suspected carpal tunnel syndrome. Am J Med Rehabil, 2002, 81: 597-600.

31) Irvine J, Chong SL, Amirjani N, et al.: Double-blind randomized controlled trial of low-level laser therapy in carpal tunnel syndrome. Muscle Nerve, 2004, 30: 182-187.

32) Fernández-de-Las-Peñas C, Pérez-de-Heredia-Torres M, Martínez-Piédrola $\mathrm{R}$, et al.: Bilateral deficits in fine motor control and pinch grip force in patients with unilateral carpal tunnel syndrome. Exp Brain Res, 2009, 194: 29-37. 